\title{
COVID-19 among HIV-infected population
}

\author{
Dian Daniella ${ }^{1}$, Alfred Setyono ${ }^{2}$, Marianto Marianto ${ }^{1}$ \\ ${ }^{1}$ Department of Internal Medicine, Wangaya Regional General Hospital, Denpasar, Indonesia \\ ${ }^{2}$ Department of Dermatology, Wangaya Regional General Hospital, Denpasar, Indonesia
}

\begin{abstract}
Novel coronavirus disease 2019, COVID-19, has been declared as a pandemic on March 11 ${ }^{\text {th }}, 2020$. The devastating impact of COVID-19 has affected every sectors and individuals worldwide. No definite and specific treatment has been discovered up till now, apart from symptomatic or experimental treatments. Individuals with chronic disease comorbidity are identified to have worse outcomes and greater risk of mortality. Moreover, certain chronic infectious disease, such as human immunodeficiency virus (HIV), is also included as affected individuals by COVID-19 with unknown extent. Various recent studies suggested that there is no higher risk of COVID-19 among HIV-infected population, but subjects are limited. Additionally, controversies still exist regarding the outcome of COVID-19 in HIVinfected population as well as the role of antiretroviral therapy (ART) in COVID-19. Another problems regarding COVID-19 pandemic in this context impede medical access and disturb adherence to ART in HIV patients, further increasing risk of lethal morbidity and mortality of COVID-19. Because of the urgency of COVID-19 pandemic as well as chronicity, mortality, and great number of HIV infections, clinicians should be aware of the relationship between HIV infection and COVID-19. This review article summarized the association between HIV infection and COVID-19 during the pandemic, including the current evidence of ART in COVID-19.
\end{abstract}

HIV AIDS Rev 2021; 20, 4: 231-234 DOI: https://doi.org/10.5114/hivar.2021.111534

Key words: HIV, AIDS, COVID, SARS-CoV-2, co-infection.

\section{Introduction}

Coronavirus disease, COVID-19, was declared as a pandemic on March $11^{\text {th }}, 2020$. The World Health Organization (WHO) and Center for Disease Control and Prevention (CDC) have issued health alerts and prevention guidelines for people worldwide, including social distancing, masks wearing, and home-base working. Up to July 2020, the novel coronavirus has affected 12,102,328 people, with 551,046 related deaths. Chronic disease comorbidities are associated with higher COVID-19 mortality, including asthma, chronic lung disease, diabetes, cardiovascular conditions, obesity, chronic kidney disease, chronic liver disease, and immunocompromised state, such as human immunodeficiency virus (HIV) [1].
It is estimated that HIV affected 37.9 million people [2], with 1.7 million newly infected cases and 690,000 deaths from acquired immunodeficiency syndrome (AIDS)-related illnesses in 2019 worldwide. HIV/AIDS is a still huge health burden in the world, and among infected people, only 25.4 million individuals have access to antiretroviral therapy (ART) [3].

$\mathrm{HIV}$ is causing abnormal humoral and T cell-mediated immune responses, resulting in increased susceptibility to numerous infections [1]. In a cohort study with 5,630 patients, Inciarte et al. reported that the incidence of confirmed COVID-19 in HIV-infected population were rated as 107 cases per 10,000 patients, with $4 \%$ of mortality rate [4]. COVID-19 added another health burden for HIV-infected population, such as physical, emotional, and social well-being, 
which eventually delay the delivery of effective healthcare and access to HIV treatment $[3,5]$. Controversies still exist regarding the risk of COVID-19 in HIV, as several other studies found the role of anti-HIV therapy as debatable [6].

Given the urgency of COVID-19 pandemic and chronicity, mortality, and great number of HIV infections, clinicians should be aware of the relationship between HIV infection and COVID-19. In this review, both COVID-19 and HIV were discussed thoroughly.

\section{Risk of COVID-19 in HIV-infected population}

So far, there is a limited number of studies regarding COVID-19 among HIV-infected population. Two published studies by Vizcarra et al. and Guo et al. had drawn our attention [7, 8]. Vizcarra et al. in Madrid had observed 51 out of 2,873 regularly controlled HIV-infected individuals, who had been either clinically or laboratory confirmed as COVID-19 up till April 30 ${ }^{\text {th }}, 2020$. Thirty-five out of 51 HIV-infected patients had been confirmed by laboratory as COVID-19-infected. This resulted in an overall 1.8\% incidence rate and $1.2 \%$ laboratory test confirmed incidence rate among HIV-infected population. During the same period, general population in Madrid had reported 269,417 cases of COVID-19, with 61,577 having laboratory test-confirmed. This accounted for $4.02 \%$ of overall incidence rate and $0.92 \%$ of laboratory test-confirmed cases from general population. These numbers showed that general population had a higher overall COVID-19 diagnosis (4.02\%) compared to HIV-infected population diagnosed with COVID-19 (1.8\%). However, infection rate becomes almost similar, if only laboratory test-confirmed cases are considered, as general population had $0.92 \%$ infection rate, compared with $1.2 \%$ of HIV-infected population [7].

At the beginning of the outbreak in Wuhan, Guo et al. has found 12 clinically diagnosed cases with 8 laboratory test-confirmed COVID-19 from 1,174 HIV-infected individuals. All the 8 patients were undergoing routine ART. This accounts for $1.02 \%$ overall infection rate and $0.68 \%$ laboratory test-confirmed COVID-19 among HIV-infected individuals. Within the similar period, the Wuhan general population recorded about 47,000 infection cases from 9 million people, with no exact data available concerning the number of laboratory test-confirmed cases, which accounts for $0.52 \%$ of general infection rate. Considering the numbers between laboratory test-confirmed HIV-infected cases with COVID-19 and non-HIV-infected individuals, it was suggested that HIV infection may not be a risk factor for COVID-19 infection [8].

The risk factor for COVID-19 among HIV-infected patients are comparable to general population. Vizcarra et al. reported on $51 \mathrm{HIV}$-infected individuals with a mean age of 53.3 ( \pm 9.5 ) years old, out of which, 43 patients $(84 \%)$ were males and 32 patients (63\%) had at least one comorbidity. Guo et al. also supports this finding, with $7 \mathrm{HIV}$-infected male individuals $(88 \%)$ with mean age of $57( \pm 9.5)$ years old $[7,8]$. Data from these studies present similar outcomes regarding COVID-19 infection rates between HIV-infected population and general population.

Other concerning issue is how CD4+ cell counts may affect the chance of COVID-19. Vizcarra et al. in their study did not find any association between nadir CD4+ cell counts and COVID-19 [7]. In contrast, multi-center study conducted by Dandachi et al. found that lower CD4+ counts was associated with higher hospitalization rates and lower survival [9]. As in general population, low CD4+ counts are also more common in severe COVID-19 cases $[10,11]$. Currently, studies assessing the risk of COVID in low CD4+ patient are lacking.

\section{HIV as severe outcome/mortality risk factor in COVID-19}

Based on the previous recent studies, COVID-19 morbidity and mortality rates among HIV-infected individuals might just be comparable to the general population. Vizcarra et al. recorded $38 \mathrm{HIV}$-infected patients with mild or moderate symptoms, and $13 \mathrm{HIV}$-infected individuals with severe symptoms. Two patients from the latter group eventually died, accounting for $15 \%$ of mortality rate. This was lower compared to general Madrid's population with severe infection mortality rate as around $20 \%$. However, it was noted that $25 \% \mathrm{HIV}$-infected individuals in the studies with HIV-infected patients with COVID-19, developed severe symptoms, with $12 \%$ of them admitted to intensive care unit (ICU). This is significantly higher than the general population, in which only $17-21 \%$ of patients developed severe symptoms, with $3-5 \%$ of them needing ICU care. This may suggest that HIV-infected individuals with COVID-19 may have higher morbidity compared to the general population [7].

Low CD $4+$ cell counts $\left(<200\right.$ cells $\left./ \mathrm{mm}^{3}\right)$ without ART is speculated to be a contributing factor for more severe form of COVID-19 in HIV-infected population [4]. In a study conducted by Guo et al., 6 of $8 \mathrm{HIV}$-infected individuals had a CD $4+$ count $>350 / \mu l$, and 2 had a CD $4+$ count ranging between 101 and 350/ $\mu$ l. Interestingly, 41 AIDS patients with CD4 $+<100 / \mu \mathrm{l}$ reported no symptoms of COVID-19. All 8 HIV-infected individuals with high CD4+ count, had viral load of $<20$ copies/ml, and 41 COVID-19 symptom-free AIDS patients had viral load of $>20$ copies $/ \mathrm{ml}$. Although it is still uncertain, previous studies suggested that immunosuppression and low CD4+ levels might spare HIV-infected patients with COVID-19 from developing cytokine storm observed in COVID-19 [4]. However, these studies are small in sample size, and further larger studies may be needed.

It was previously suggested that lower CD4+ counts in HIV-infected individuals may result in better outcomes if infected with SARS-CoV-2 due to less possibility of cytokine storm development $[6,8,12]$. Nonetheless, this was also based on studies from 2003 SARS-CoV and 2015 Middle East respiratory syndrome (MERS-CoV) outbreaks, which suggested that HIV-infected patients often had lower risk 
of infection or progression to severe disease. This might happen because of coronavirus replication was inhibited by ART, but the immunosuppressive state of patients resulted in longer recovery time required than non-HIV-infected population [6].

While it is yet to be fully explored, Vizcarra et al. study found that among a severe disease group subjects, most of them presented with a lower lymphocyte count, and 4 out of 6 HIV-infected individuals (67\%) in ICU care had CD4+ levels of less than 200/ $\mu$ l [7]. Interestingly, Guo et al. study showed all $8 \mathrm{HIV}$-infected individuals with higher CD4+ levels, 2 (25\%) with $101-350 / \mu \mathrm{l}$, and $6(75 \%)$ with $>350 / \mu \mathrm{l}$. All the $8 \mathrm{HIV}$-infected individuals also recorded HIV viral load of less than 20 copies/ml [8]. These two different results have not been able to suggest how CD4+ levels are associated with patients' outcomes. This needs to be confirmed with more studies in the future, with larger sample size. However, it is recommended to administer a prophylaxis treatment to prevent opportunistic infection among HIV-infected individuals presenting with low CD4+ levels [13].

Guo et al. did not specify on comorbidities, but Vizcarra et al. discovered the prevalence of hypertension, obesity, diabetes, chronic kidney disease, and chronic liver disease as higher compared to SARS-CoV-2 or HIV alone. While comorbidities still pose as a risk factor for all population, this study suggest the risk of severe outcomes can be higher for HIV-infected individuals [7]. A previous systematic review has also suggested that HIV-infected patients with COVID-19 tend to be accompanied by multiple morbidities, more severe disease, and higher mortality rate [1]. Additionally, a cohort study from Africa found HIV infection associated with doubling mortality risk of COVID-19 [14].

\section{ART effects on COVID-19}

Currently, WHO guidelines indicate dual nucleoside/ nucleotide reverse transcriptase inhibitors (NRTI) combined with non-nucleoside reverse transcriptase inhibitors (NNRTI) or protease inhibitor (PI), or integrase inhibitor as recommended treatments. Tenofovir disoproxil fumarate (TDF), lamivudine, and efavirenz are preferred as the first line ART in adult patients. In some cases, zidovudine can be used as an alternative of tenofovir [15].

In COVID-19 individuals, a variety of ART drugs are being considered as treatment of the disease. Proteinase inhibitors (lopinavir-ritonavir) inhibit enzymes that activate envelope glycoproteins as a part of viral entry into cells, which may work in COVID-19 [16]. Lopinavir-ritonavir $(\mathrm{LPV} / \mathrm{r})$ was also demonstrated to show promising results in vitro [17] and animal model [18] activity against MERSCoV. However, in a randomized, controlled, open-label trial involving hospitalized adult patients with confirmed SARS-CoV-2 infection, investigating the efficacy and safety of $\mathrm{LPV} / \mathrm{r}$, the addition of $\mathrm{LPV} / \mathrm{r}$ treatment to standard supportive care was not associated with clinical improvement or mortality in seriously ill patients with COVID-19 [19].
In molecular studies, TDF has been recently shown to bind to SARS-CoV-2 RNA polymerase (RdRp), with significant binding energies to a similar extent as remdesivir. Therefore, TDF has been suggested as one of the potential treatments for COVID-19 [20]. In a previous study conducted by Amo et al., the risk of confirmed cases of COVID-19 in HIV-infected individuals receiving TDF/emtricitabine (TDF/3TC)-based regimen was lower compared to other regimens [21]. Tenofovir disoproxil fumarate was found to suppress the production of inflammatory cytokines, such as interleukin (IL)-8 and IL-10. However, in the present study, there was less intensive testing for SARS-CoV-2 infection in TDF/3TC group compared with those receiving other ART regimens [21]. Also, the study did not examine confounding comorbidities. On the other hand, case series by Harter et al. and Byrd et al. found different results, in which TDF did not show protective effect against COVID-19 [22, 23].

As in the studies conducted by Vizcarra et al. and Guo et al., routine ART (consisted of NNRTI and NRTI) may not suggest any additional protection from COVID-19. In each study, all 51 and $8 \mathrm{HIV}$-infected individuals, respectively, were under regular ART. However, this could not be an accurate representation, as the authors only observed HIVinfected individuals who mostly had already undergone ART.

Since there are a lot of asymptomatic COVID-19 cases, it was possible that unknown HIV-infected individuals contracting COVID-19 were not included in these studies, as majority of COVID-19 testing to all HIV-infected patients was not available $[7,8]$.

Even though higher mortality rate due to COVID-19 was reported in immunosuppressed patients, HIV infection was not identified as significant comorbid condition in COVID-19 in several previous reports, especially HIV patients under regular ART [24-27]. People living with HIV who are on regular and effective treatment, were considered to have the same risk for COVID-19 as the general population [28]. Prior studies found that HIV-infected individuals can present with a lower risk of COVID-19 [6]. Antiretroviral therapy may reduce the risk of COVID-19. Some NRTI used as anti-HIV therapy can inhibit SARS-CoV-2 RNA-dependent RNA polymerase [4]. Currently, two large randomized clinical trials in Spain and Colombia were investigating $\mathrm{TDF} / 3 \mathrm{TC}$ regarding its' effectiveness in preventing transmission of COVID-19 [29,30]. Other protease inhibitor, darunavir/cobicistat is also under investigation in a clinical trial exploring its' effectiveness in treatment of COVID-19 [31].

\section{Conclusions}

Data from recent studies found no significant differences regarding COVID-19 infection rates between HIV-infected individuals and the general population. Lower CD4+ counts in HIV may be associated with higher hospitalization rate and lower survival. HIV presented with comorbidities may be at higher risk of developing more severe symptoms. However, larger studies are needed to further investigate the impact of HIV in COVID-19. Various ART treatments 
are still on trial regarding their effectiveness in COVID-19. The COVID-19 pandemic has limited medical access and disrupted adherence to ART on HIV. Healthcare centers must readily adapt to the situation to avoid poor outcomes, especially in vulnerable patients since individuals who are unaware of their HIV diagnosis and having limited access to healthcare, may be at increased risk of lethal morbidity and mortality of COVID-19.

\section{Conflict of interest}

The authors declare no conflict of interest.

\section{References}

1. Mirzaei H, McFarland W, Karamouzian M, Sharifi H. COVID-19 among people living with hiv: a systematic review. AIDS Behav 2021; 25: 85-92.

2. Karmen-Tuohy S, Carlucci PM, Zervou FN, et al. Outcomes among HIV-positive patients hospitalized with COVID-19. J Acquir Immune Defic Syndr 2020; 85: 6-10.

3. UNAIDS.GlobalHIV statistics: endingtheAIDSepidemic.UNAIDS; 2019.

4. Inciarte A, Gonzalez-Cordon A, Rojas J, et al. Clinical characteristics, risk factors, and incidence of symptomatic coronavirus disease 2019 in a large cohort of adults living with HIV: a single-center, prospective observational study. AIDS 2020; 34: 1775-1780.

5. Shiau S, Krause KD, Valera P, Swaminathan S, Halkitis PN. The burden of COVID-19 in people living with HIV: a syndemic perspective. AIDS Behav 2020; 24: 2244-2249.

6. Xu Z, Zhang C, Wang FS. COVID-19 in people with HIV. Lancet HIV 2020; 7: e524-526.

7. Vizcarra P, Pérez-Elías MJ, Quereda C, et al. Description of COVID19 in $\mathrm{HIV}$-infected individuals: a single-centre, prospective cohort. Lancet HIV 2020; 7: e554-564.

8. Guo W, Ming F, Dong Y, et al. A survey for COVID-19 among HIV/AIDS patients in two districts of Wuhan, China. SSRN Electron J [Internet]; 2020. Available from: https://www.ssrn.com/abstract=3550029 (Accessed: 06.10.2020).

9. Dandachi D, Geiger G, Montgomery MW, et al. Characteristics, comorbidities, and outcomes in a multicenter registry of patients with HIV and coronavirus disease-19. Clin Infect Dis 2021; 73: e1964-e1972.

10. Liu Z, Long W, Tu M, et al. Lymphocyte subset (CD4+, CD8+) counts reflect the severity of infection and predict the clinical outcomes in patients with COVID-19. J Infect 2020; 81: 318-356.

11. Zhang H, Wu T. CD4+T, CD8+T counts and severe COVID-19: a meta-analysis. J Infect 2020; 81: e82-e84.

12. Gervasoni C, Meraviglia P, Riva A, et al. Clinical features and outcomes of patients with human immunodeficiency virus with COVID-19. Clin Infect Dis 2020; 71: 2276-2278.

13. EACS (European AIDS Clnical Society) and BHIVA. Statement on risk of COVID-19 for people living with HIV (EACS \& BHIVA) [Internet]; 2020. Available from: https://www.eacsociety.org/home/ covid-19-and-hiv.html (Accessed: 04.10.2020).

14. Davies MA. HIV and risk of COVID-19 death: a population cohort study from the Western Cape Province, South Africa. MedRxiv 2020. doi: 10.1101/2020.07.02.20145185. Preprint.

15. Beyrer C. Consolidated guidelines on HIV prevention, diagnosis, treatment and care for key populations [Internet]. Place of publication not identified: World Health Organization; 2016. Available from: http://proxy.library.carleton.ca/loginurl=https://www.deslibris.ca/ID/10063272 (Accessed: 05.10.2020).
16. Zhou Y, Vedantham P, Lu K, et al. Protease inhibitors targeting coronavirus and filovirus entry. Antiviral Res 2015; 116: 76-84.

17. de Wilde AH, Jochmans D, Posthuma CC, et al. Screening of an FDA-approved compound library identifies four small-molecule inhibitors of Middle East respiratory syndrome coronavirus replication in cell culture. Antimicrob Agents Chemother 2014; 58: 4875-4884.

18. Chan JFW, Yao Y, Yeung ML, et al. Treatment with lopinavir/ritonavir or interferon- $\beta 1 \mathrm{~b}$ improves outcome of MERS-CoV infection in a nonhuman primate model of common marmoset. J Infect Dis 2015; 212: 1904-1913.

19. Cao B, Wang Y, Wen D, et al. A trial of lopinavir-ritonavir in adults hospitalized with severe COVID-19. N Engl J Med 2020; 382: 1787-1799.

20. Elfiky AA. Ribavirin, remdesivir, sofosbuvir, galidesivir, and tenofovir against SARS-CoV-2 RNA dependent RNA polymerase (RdRp): a molecular docking study. Life Sci 2020; 253: 117592.

21. Del Amo J, Polo R, Moreno S, et al. Incidence and severity of COVID-19 in HIV-positive persons receiving antiretroviral therapy: a cohort study. Ann Intern Med 2020; 173: 536-541.

22. Härter G, Spinner CD, Roider J, et al. COVID-19 in people living with human immunodeficiency virus: a case series of 33 patients. Infection 2020; 48: 681-686.

23. Byrd KM, Beckwith CG, Garland JM, et al. SARS-CoV-2 and HIV coinfection: clinical experience from Rhode Island, United States. J Int AIDS Soc [Internet]; 2020. Available from: https://onlinelibrary.wiley.com/doi/abs/10.1002/jia2.25573 (Accessed: 05.10.2020).

24. Richardson S, Hirsch JS, Narasimhan M, et al. Presenting characteristics, comorbidities, and outcomes among 5700 patients hospitalized with COVID-19 in the New York City area. JAMA 2020; 323 : 2052-2059.

25. Borobia A, Carcas A, Arnalich F, et al. A cohort of patients with COVID-19 in a major teaching hospital in Europe. J Clin Med 2020; 9: 1733.

26. Ridgway JP, Farley B, Benoit JL, et al. A case series of five people living with HIV hospitalized with COVID-19 in Chicago, Illinois. AIDS Patient Care STDs 2020; 34: 331-335.

27. Marimuthu J, Kumar BS, Aravind Gandhi P. HIV and SARS CoV-2 co-infection: a retrospective, record based, case series from South India. J Med Virol 2021; 93: 163-165.

28. National Institute of Health. Interim Guidance for COVID-19 and Persons with HIV. In 2020. Available from: https://clinicalinfo.hiv. gov/en/guidelines/covid-19-and-persons-hiv-interim-guidance/ interim-guidance-covid-19-and-persons-hiv?view=full.

29. Daily Regimen of Tenofovir/Emtricitabine as Prevention for COVID-19 in Health Care Personnel in Colombia [Internet]. Available from: https://clinicaltrials.gov/ct2/show/NCT04519125 (Accessed: 04.10.2010).

30. Randomized Clinical Trial for the Prevention of SARS-CoV-2 Infection (COVID-19) in Healthcare Personnel (EPICOS) [Internet]. Available from: https://clinicaltrials.gov/ct2/show/NCT04334928 (Accessed: 03.10.2010).

31. Efficacy and Safety of Darunavir and Cobicistat for Treatment of COVID-19 (DC-COVID-19) [Internet]. Available from: https:// clinicaltrials.gov/ct2/show/NCT04252274 (Accessed: 04.10.2010). 